\section{Europäische Umfrage zu ADHS}

— Weltweit haben 5,3\% der Schulkinder eine Aufmerksamkeitsdefizit-/Hyperaktivitätsstörung (ADHS). Bei mindestens einem Drittel bleiben die Probleme im fortgeschrittenen Alter bestehen. Erste Ergebnisse einer europäischen Umfrage belegen die weitreichenden Beeinträchtigungen der Betroffenen sowie Defizite in der medizinischen Versorgung: Im Vergleich zu Kindern ohne ADHS weisen gemäß der Online-Befragung in sechs europäischen Ländern Kinder mit ADHS insgesamt signifikante Beeinträchtigungen auf $(p<0,001)$. Diese betrafen alle abgefragten Lebensbereiche wie Schule, Sozialkontakte, Familie, Freundschaften und das Verhältnis zu Geschwistern und Eltern. Darüber hinaus gehörten die Betroffenen bei den Schulleistungen häufiger zum unteren Drittel der Klasse. Kinder mit ADHS würden zudem seltener eingeladen und hätten weniger soziale Kontakte; sie fühlten sich häufiger frustriert und handelten öfter unüberlegt, betonte Dr. Hervé Caci, Nizza/Frankreich, der die Daten erstmals in Berlin vorstellte. Die Befragung basiert auf rund 400 Teilnehmern pro Land: je 100 Eltern oder Erziehungsberechtigte von Kindern mit bzw. ohne ADHS sowie je 100 Erwachsene mit bzw. ohne ADHS. Wie sich weiter zeigte, dauert es im Durchschnitt 26,8 Monate, bis die
Diagnose ADHS gestellt wird. $38 \%$ der Eltern hätten bis dahin mehr als drei Ärzte aufgesucht, hob Caci hervor. $46 \%$ der Kinder mit ADHS erhielten eine evidenzbasierte Pharmakotherapie, mit der $73 \%$ ihrer Eltern zufrieden waren. Die Daten zu den Erwachsenen werden laut Caci im Herbst präsentiert. Um die Versorgungssituation von ADHSPatienten in Deutschland zu verbessern, hat das Unternehmen Shire dieses Jahr eine Informations-Kampagne initiiert (www. adhs-zukunftstraeume.de). Dr. Petra Eiden

Journalisten-Workshop „ADHD in Europe: charting new territory - erste Ergebnisse des europäischen Lifetime Impairment Survey". Berlin, 27. Mai 2011. Veranstalter: Shire Deutschland

\title{
Primäre Immundefekte früh erkennen und behandeln
}

Häufig dauert es Jahre bis ein primärer Immundefekt (PID) entdeckt wird. Wir befragten Prof. Dr. Volker Wahn aus Berlin.

? Bei den PID unterscheidet man zwischen mindestens 170 genetisch determinierten Formen. Warum gibt es keine verlässlichen Zahlen zur Prävalenz?

Wahn: Diese Angabe basiert noch auf der Klassifikation des IUIS-Reports von 2009. Die Anzahl der primären Immundefekte wächst ständig. Heute geht man schon von rund 200 verschiedenen genetisch determinierten Formen aus. Nach den Ergebnissen einer Telefonumfrage in den USA wurde eine Prävalenz symptomatischer Immundefekte von $1: 2.000$ ermittelt. Nimmt man hingegen die aktuelle Anzahl der diagnostizierten Patienten in Deutschland, die bei etwa 4.000 liegt, erhält man einen Wert von circa $1: 20.000$. Die Wahrheit liegt wahrscheinlich zwischen diesen beiden Zahlen. Ich schätze die Prävalenz der symptomatischen primären Immundefekte in Deutschland auf 1:5.000.

? Ist die Diagnose tatsächlich so schwierig? Wahn: Ja, das ist durchaus nicht einfach. Die Schwierigkeit liegt darin, dass eine Sinusitis oder eine Pneumonie nicht unbedingt auf einen Immundefekt hinweisen. Zum Beispiel geben erst eine auffällige Häufung dieser Erkrankungen oder das Auftreten bestimmter Erreger, die bei einem gesunden Immunsy- stem nicht auftreten sollten, entsprechende Hinweise. Hier sollte dann über eine genauere Diagnostik, wie ein Differenzialblutbild zusammen mit der quantitativen Bestimmung von IgG, IgM und IgA, oder sogar der IgG-Subklassen, nachgedacht werden. Bei schwerwiegenden Infektionen kann auch eine direkte Überweisung des Patienten in ein Immundefektzentrum erforderlich sein.

? Wo sehen Sie Optimierungsbedarf bei der Diagnostik?

Wahn: Optimierungsbedarf sehe ich im Bewusstsein der Ärzte. Viele Kollegen in Krankenhäusern und Praxen sind noch nicht dafür sensibilisiert, selbst beim Vorliegen von eindeutigen Hinweisen, an Immundefekte zu denken. Eine frühzeitige Diagnosestellung oder eine Überweisung in ein Schwerpunktzentrum für primäre Immundefekte kann den Betroffenen häufig einen langen Leidensweg ersparen. Um diesen Prozess zu unterstützen, habe ich zusammen mit Professor Tim Niehues (HELIOS-Kliniken, Krefeld) und Gabriele Gründl (Deutsche Selbsthilfe Angeborene Immundefekte e.V., dsai) die Netzwerkinitiative FIND-ID entwickelt. Mit deren Hilfe soll die „Awareness" für primäre Immundefekte bei allen Arztgruppen durch gezielte Aktionen und Informationen erhöht werden.

? Seit kurzem ist mit Hizentra ${ }^{\circledR}$ ein neues 20\%iges Immunglobulin auf dem Markt. Worin sehen Sie die Vorzüge des Produktes?

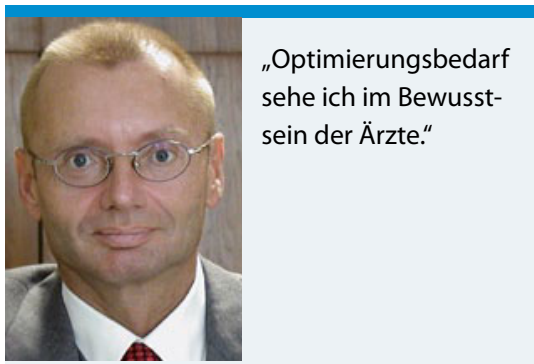

Prof. Dr. med. Volker Wahn Oberarzt und Leiter der Sektion Infektionsimmunologie Charité - Universitätsmedizin Berlin, Mitbegründer der Initiative Find-ID

Wahn: Die Ergebnisse der Zulassungsstudien zu Hizentra ${ }^{\circledast}$ in den USA und in Europa zeigen, dass die anti-infektiöse Wirkung von Hizentra ${ }^{\circledast}$ sehr gut ist. Auch die Verträglichkeit und das Nebenwirkungsprofil sind gut. Im Gespräch mit Patienten stellt sich vor allem die deutlich verkürzte Infusionszeit als ein großer Vorteil des Produkts dar. Dieser resultiert aus der höheren IgG-Konzentration und der zugelassenen Infusionsgeschwindigkeit von $25 \mathrm{ml} / \mathrm{h} / \mathrm{Infusionsstelle.} \mathrm{Beson-}$ ders aktive Patienten, die noch im Berufsleben stehen, und solche, die hohe Dosen benötigen, profitieren davon. Von Vorteil ist auch die durchgängige Lagerbarkeit bei Raumtemperatur durch die Stabilisierung mit L-Prolin - besonders im Urlaub oder auf Reisen müssen Patienten sich keine Sorgen mehr um die Kühlung machen.

Nach Informationen von CSL Behring, Hattersheim 